\title{
Perfil nutricional de idosos internados nas enfermarias de clínica médica de um hospital da Amazônia
}

\author{
Nutritional profile of elderly people in nurses of medical clinic of an Amazon hospital
}

Perfil nutricional de idosos internados en las enfermarias de clínica medica de un hospital de Amazonia

Rudival Faial De Moraes Junior ${ }^{1}$, Marina Maria Guimarães Borges ${ }^{1}$, Andrea Negrão Costa ${ }^{1}$, Elis Barros Begot ${ }^{1}$, Ana Carolina Alves Marques De Souza ${ }^{1}$, Carla Daniele Nascimento Pontes ${ }^{1 *}$, Lucianna Serfaty De Holanda ${ }^{1}$.

\section{RESUMO}

Objetivo: Determinar o estado nutricional dos pacientes idosos internados em um hospital da Amazônia através da aplicação da Mini Avaliação Nutricional (MAN). Metodologia: Trata-se de um estudo observacional, transversal e descritivo. A pesquisa foi realizada nas Enfermarias de clínica médica da Fundação Santa Casa de Misericórdia do Pará (FSCMPA). A população estudada foi constituída pelos idosos internados nas enfermarias acima citadas, no período de junho a agosto de 2017. Resultados: Dos 28 pacientes estudas $57,1 \%$ apresentavam menos de 70 anos. A grande maioria dos pacientes avaliados $(82,1 \%)$ foi classificada como possível desnutrição no escore de controle, sendo estes $60,9 \%$ com risco de desnutrição de fato e 34,8\% fecharam diagnóstico de desnutrição. Conclusão: A aplicação da MAN em idosos internados, bem como ambulatoriais é de extrema importância para avaliar os riscos e realizar intervenção precocemente, prevenir possíveis agravantes, diminuir o tempo de internação ou evitar a mesma, melhorar o prognostico do paciente e aumentar chance de recuperação.

Palavras-Chave: Estado Nutricional, Mini-Analiação Nutricional, Idosos.

\section{ABSTRACT}

Objective: To determine the nutritional status of hospitalized patients in an Amazonian hospital through the Mini Nutritional Assessment (MAN). Methodology: This is an observational, transversal and descriptive study. The research was carried out in the Medical Clinic Nursing of the Santa Casa de Misericordia Foundation of Pará (FSCMPA). The study population consisted of the elderly hospitalized in the aforementioned wards from June to August 2017. Results: Of the 28 patients studied, $57.1 \%$ were younger than 70 years. The majority of the patients evaluated $(82.1 \%)$ were classified as possible malnutrition in the control score, being these $60.9 \%$ with a risk of malnutrition in fact and $34.8 \%$ were diagnosed as malnourished. Conclusion: The application of MAN to hospitalized elderly as well as outpatients is extremely important in order to evaluate risks and perform early intervention, to prevent possible aggravation, to reduce hospitalization time or to avoid hospitalization, to improve the patient's prognosis and to increase the chance of recovery.

Key Words: Nutritional Status, Nutritional Mini-Analiation, Elderly.

\footnotetext{
${ }^{1}$ Fundação Santa Casa de Misericórdia do Pará. * E-mail: pontes4@live.com
} 


\section{RESUMEN}

Objetivo: Determinar el estado nutricional de los pacientes internados en un hospital de la Amazonía a través de la aplicación de la MiniAvaluación Nutricional (MAN). Metodología: Se trata de un estudio observacional, transversal y descriptivo. La investigación fue realizada en las Enfermerías de clínica médica de la Fundación Santa Casa de Misericordia del Pará (FSCMPA). La población estudiada fue constituida por los ancianos internados en las enfermerías arriba citadas, en el período de junio a agosto de 2017. Resultados: De los 28 pacientes estudiados $57,1 \%$ presentaban menos de 70 años. La gran mayoría de los pacientes evaluados $(82,1 \%)$ fue clasificada como posible desnutrición en el score de control, siendo estos $60,9 \%$ con riesgo de desnutrición de hecho y el 34,8\% cerró diagnóstico de desnutrición. Conclusíon: La aplicación de la MAN en ancianos internados, así como ambulatorios es de extrema importancia para evaluar los riesgos y realizar intervención precozmente, prevenir posibles agravantes, disminuir el tiempo de internación o evitar la misma, mejorar el pronóstico del paciente y aumentar la posibilidad de recuperación.

Palabras Clave: Estado Nutricional, Mini-Anhalación Nutricional, Ancianos.

\section{INTRODUÇÃO}

A população idosa vem crescendo ao longo dos anos e há uma projeção que estima que em 30 anos a quantidade total de idosos possa dobrar. Porém, juntamente com esse crescimento populacional há um aumento de problemas relacionados ao envelhecimento como doenças crônicas e incapacidades, para as quais os familiares e profissionais de saúde precisam estar preparados (MIRANDA et al 2016).

O envelhecimento populacional trouxe modificações na expectativa de vida e preocupações quanto à qualidade de vida da população idosa. Os estudos epidemiológicos sobre o estado nutricional em idosos indicam que os distúrbios nutricionais estão associados com o risco de morbidade e de mortalidade (SILVEIRA,2009).

Os estudos mostram que a desnutrição tem se apresentado com alta prevalência em idosos. Os valores oscilam de 15 a $60 \%$, dependendo do local onde o idoso se encontra - em casa, asilo ou hospital - e da técnica utilizada para diagnóstico de desnutrição. A avaliação da nutrição geriátrica tem uma grande importância visto que, nesta fase, há diversas implicações nutricionais, que variam desde desnutrição proteico calórica, alterações metabólicas, interações medicamentosas, até deficiência de micronutrientes, que associadas a fatores psicológicos, sociais e econômicos, podem contribuir para o declínio e comprometimento de várias funções orgânicas que são vitais para o indivíduo idoso (CAMPOS et al 2006).

O idoso apresenta modificações fisiológicas como redução de reservas funcionais, redução da quantidade proteica e aumento do tecido adiposo e devido a isso os apresenta tendência a desenvolver desnutrição. Todos os sistemas orgânicos são afetados pelo envelhecimento, levando a uma redução global do funcionamento e das reservas. Como fatores de risco para desnutrição podemos destacar dessas alterações a redução da massa magra, aumento da gordura corporal, aumento do turn-over ósseo e redução das fibras musculares (FELIX e SOUZA 2009).

Assim, a deficiência nutricional é um problema relevante nessa população, decorrente de alterações do próprio envelhecimento, doenças sistêmicas e/ou situação socioeconômica, que condicionam o seu estado nutricional. Uma avaliação nutricional simples e sistematizada, que permita detectar precocemente e de maneira simples os idosos em risco nutricional, para uma posterior avaliação completa dos mesmos, deveria fazer parte de forma protocolizada da assistência ao paciente geriátrico institucionalizado (ABAJO, 2008).

O ambiente hospitalar favorece o aparecimento de desnutrição devido a uma ingesta alimentar reduzida frente a uma necessidade aumentada, uma vez que os pacientes hospitalizados frequentemente possuem redução do apetite dificultando a alimentação e possuem demanda metabólica aumentada para combater o agravo que causou hospitalização (BARBOSA-SILVA e BARROS, 2002). 
Portanto, a avaliação nutricional admissional vem ganhando cada vez mais importância nesse contexto, a fim de identificar precocemente o paciente desnutrido para trata-lo durante a internação e para identificar os pacientes com maior risco de desenvolver desnutrição, a fim de evita-la (BRAGA e SERRANO, 2007).

Dessa forma, este estudo tem por objetivo determinar o estado nutricional dos pacientes idosos internados em um hospital da Amazônia através da aplicação da Mini Avaliação Nutricional (MAN).

\section{METODOLOGIA}

Trata-se de um estudo observacional, transversal e descritivo. A pesquisa foi realizada nas Enfermarias de clínica médica da Fundação Santa Casa de Misericórdia do Pará (FSCMPA). A população estudada foi constituída pelos idosos internados nas enfermarias acima citadas, no período de junho a agosto de 2017. A amostra foi constituída seguindo os critérios de inclusão e exclusão.

A coleta de dados foi feita pela análise dos parâmetros estabelecidos pela Mini avaliação nutricional (MAN) do idoso e protocolo com dados clínicos do paciente previamente elaborado, após aprovação do Comitê de Ética conforme parecer de número 2.929.514.

Os dados referentes à identificação, hábitos e antropometria foram obtidos após explicação e assinatura do TCLE através de uma entrevista com o idoso e/ou com seu responsável legal realizada durante a aplicação do MAN. A MAN é um formulário que contém 18 perguntas divididas entre Triagem e Avaliação Global. Inicialmente é feita uma triagem com abordagem de hábitos alimentares, perda de peso, estresse psicológico, grau de mobilidade, IMC (índice de massa corpórea) e problemas neurológicos. Já a segunda parte da MAN é realizado apenas se a triagem estiver alterada, complementando então a Avaliação global, a qual é composta por investigação quanto ao modo de vida, mobilidade, medicação, alterações psicológicos, circunferência do braço e circunferência da panturrilha, número de refeições, ingestão de alimentos e líquidos, autonomia para comer, percepção da saúde e da condição nutricional.

A partir dessa avaliação é feita a soma dos pontos obtidos na triagem somados aos pontos da avaliação global e há uma interpretação na qual pontuação acima oi igual a 24 indica bom estado nutricional, pontuação entre 17 e 24,5 indica risco de desnutrição e pontuação menor que 17 é confirmada desnutrição.

A antropometria foi composta por peso, altura e Índice de Massa Corporal foi realizada em pacientes sem calçados e sem agasalhos, vestindo apenas roupas leves, na posição em pé com os pés juntos. Foi utilizada uma mesma balança antropométrica. Todos os pacientes da presente pesquisa serão estudados segundo os preceitos da Declaração de Helsinque e do Código de Nuremberg, respeitadas as normas de pesquisa envolvendo seres humanos (Res. CNS 196/96) do Conselho Nacional de Saúde.

Foi realizada análise quantitativa através de recursos da informática, utilizando os consecutivos programas Microsoft Excel 2007 para compilação de dados e elaboração de gráficos e Microsoft Word 2007 pra digitalização de textos, bem como análise pelo Bioestat 5.0.

\section{RESULTADOS}

Este estudo avaliou um total de 28 pacientes idosos, nos quais houve um predomínio de pacientes do sexo feminino, com 53,6 \% estudados, contudo uma diferença pequena em relação aos pacientes do sexo masculino. Em relação a faixa etária, o trabalho estratificou os pacientes em 3 grupos. Os pacientes entre 6069 anos apresentaram maior número dentro de todos os indivíduos, com 16 pessoas do total de 28 pacientes inclusos, representando $57,1 \%$ dos pacientes estudados. Os pacientes com 70 a 79 anos e os com 80 anos ou mais, ambas as categorias com $21,4 \%$ dos pacientes. A maior parte dos pacientes analisados através do IMC no presente trabalho estava incluída na classificação do excesso de peso, com mais de $40 \%$ do total de indivíduos estudados. Os demais pacientes se distribuiram entre magreza (29\%) e Eutrofia (30\%) (Gráfico 1). 
Figura 1 - IMC dos pacientes.

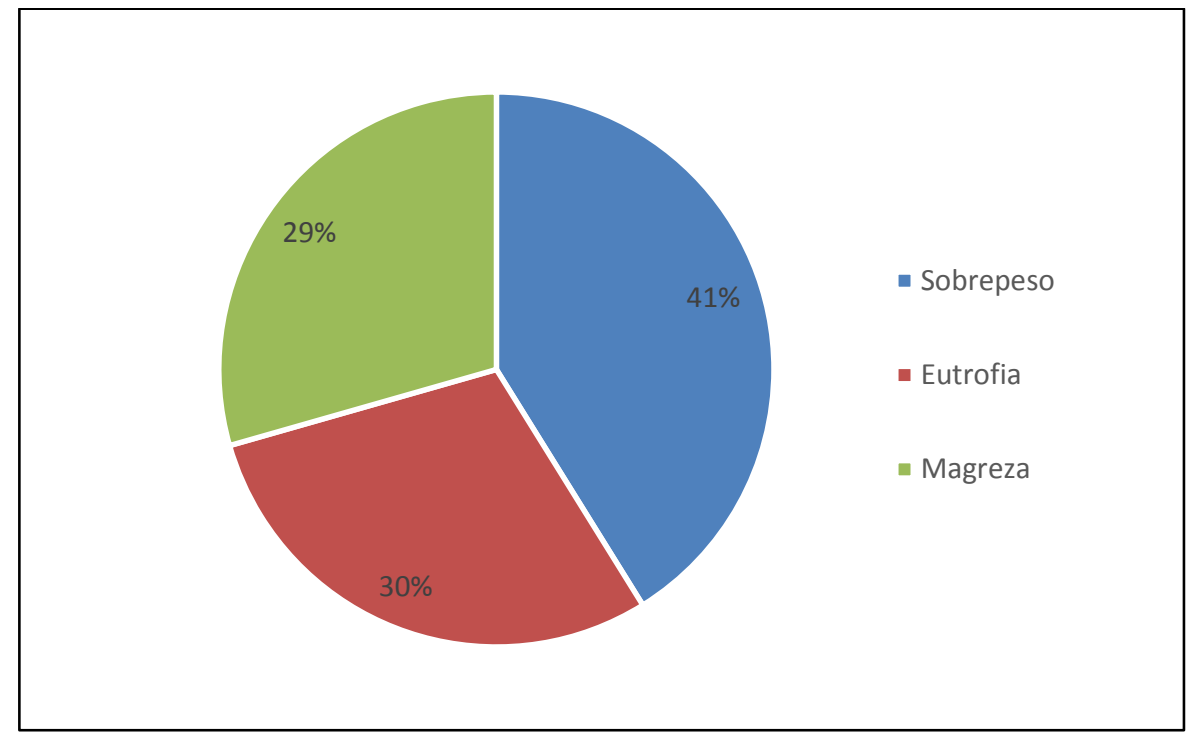

Fonte: Dados Primários.

Os pacientes analisados dentro da classificação de controle da MAN neste estudo, tiveram 23 dos seus doentes dentro de possível desnutrição, com $82,1 \%$ das pessoas estudadas. Estes resultados foram estatisticamente significativos com índice de rejeição da hipótese de nulidade tendo resultado inferior a 0,05 (Tabela 1).

Tabela 1 - Classificação de controle dos pacientes estudados conforme a triagem da MAN no período de junho a agosto de 2017 em um hospital da Amazônia.

\begin{tabular}{ccc}
\hline Classificação do controle & Freq & $\%$ \\
\hline Normal & 5 & $17,9 \%$ \\
Possível desnutrição* $^{*}$ & 23 & $82,1 \%$ \\
\hline Total & $\mathbf{2 8}$ & $\mathbf{1 0 0 , 0 \%}$ \\
\hline
\end{tabular}

${ }^{*} p<0.0001$ Teste Qui-Quadrado Aderência

Fonte: Dados Primários.

Os 23 pacientes que possuíam risco de desnutrição na triagem da MAN foram submetidos a Mini Avaliação Completa a fim de identificar quais destes possuíam deveras desnutrição. O resultado mostrou que mais de $60 \%$ deles possuía de fato risco de desnutrição, 34,8\% apresentou diagnóstico de desnutrição confirmado e somente 4,3\% não apresentaram risco de desnutrição ou desnutrição (Tabela 2). Estes resultados foram estatisticamente significativos. Neste estudo, observamos que $100 \%$ das mulheres e $91 \%$ dos homens apresentavam risco de desnutrição ou desnutrição. Dos pacientes com risco de desnutrição ou desnutrição $42,9 \%$ dos pacientes analisados estavam inclusos na faixa etária de pacientes com 70 anos ou mais, seguido da idade de maior ou igual a 80 anos com 39,3\% e pôr fim a idade de 60 a 69 anos com $17,8 \%$ dos pacientes. 
Tabela 2 - Escore do indicador de desnutrição dos pacientes que tiveram possível desnutrição no teste de triagem da MAN no período de junho a agosto de 2017 em um hospital da Amazônia.

\begin{tabular}{ccc}
\hline Escore do indicador de desnutrição & Freq & $\%$ \\
\hline Normal & 1 & $4,3 \%$ \\
Risco de desnutrição* & 14 & $60,9 \%$ \\
Desnutrição* & 8 & $34,8 \%$ \\
Total & $\mathbf{2 3}$ & $\mathbf{1 0 0 , 0 \%}$ \\
\hline${ }^{*} p<0.0001$ Teste G Aderência & &
\end{tabular}

Fonte: Dados Primários.

\section{DISCUSSÃO}

O envelhecimento é um processo natural e irreversível, o qual é acompanhado de mudanças biológicas, fisiológicas e bioquímicas que resultam em alterações sociais, psicológicas e comportamentais (BLANCO et al 2006).

Vários instrumentos de diagnostico têm sido desenvolvidos para diagnosticar e tratar pacientes de alto risco. Entre eles a MNA (Mini Nutricional Assessment) foi projetada e validada para promover uma simples e rápida avaliação do estado nutricional de pacientes idosos em clínicas, hospitais e asilos com o objetivo de avaliar o risco de desnutrição e permitir uma intervenção antecipada nutricional quando necessário (VELLAS et al)

No presente estudo a maioria da população internada constituía idosos na faixa etária entre 60-69 anos, fato este, estatisticamente significante. Muitas vezes não há por parte da população um envelhecimento saudável e uma prevenção de comorbidades, levando o indivíduo a adoecer na chegada da senescência, colaborando para internações, piora de doenças pré-existentes e déficit nutricional importante. Gomes (2008) demostrou que o envelhecimento populacional é acompanhado de diversas doenças que tem como fator de risco relevante a idade avançada.

Um estudo de morais (2008), analisando os pacientes, segundo a classificação Lipschitz, 1994 classificou como magreza $38 \%$ (20) dos pacientes, eutrofia 27\% (14) e sobrepeso $34 \%$ (18). Dados estes diferentes do presente estudo que apresentou 42,9\% (12) dos indivíduos com excesso de peso; $28,6 \%(8)$ dos pacientes com eutrofia e $28,6 \%$ (8) dos pacientes com magreza. Scatolinn (2005), em estudo sobre perfil nutricional de pacientes internados relata que a maioria dos pacientes hospitalizados depende não de uma provisão de alimentação enteral ou parenteral, ou ainda de suplementos nutricionais, mas sim da adequada provisão de comida. Contudo, a questão da nutrição é dificilmente incluída nos programas de screening de idosos internados. Este rastreio é fundamental para distinguir entre a deterioração do estado geral devido a uma patologia ou um déficit nutricional. Aliado a esta informação, temos também o exagerado consumo de alimentos ricos em carboidratos de alto índice glicêmico, calóricos e pouco nutritivos que muitas vezes esses idosos estão consumindo, o que vem corroborar com os expressivos dados de pacientes com excesso de peso e obesidade (SILVA 2000).

De acordo com a MAN, os pacientes foram classificados no escore de controle como normal ou possível desnutrição, sendo nesse estudo encontrado 17,9\% (5 pacientes) e 82,1\% (23 pacientes), respectivamente, resultado este, estatisticamente significantes. 
Nos pacientes classificados como possível desnutrição, houve continuidade da avaliação com a reclassificação dos pacientes em normal, risco de desnutrição e desnutrição, sendo encontrados $4,3 \%$ normal (1 paciente), risco de desnutrição 60,9\% (14 pacientes) e desnutrição 34,8\% (8 pacientes). De acordo com Scatollin, et al, 2005, no seu estudo houve $25 \%$ dos pacientes desnutridos, $44 \%$ em risco de desnutrição e $31 \%$ fora de risco. No estudo de Moraes et al 2010 , os pacientes também foram classificados de acordo com o MAN tendo como resultados 46\% (24 pacientes) desnutridos, 35\% (18 pacientes) em risco de desnutrição e 19\% (10 pacientes) não apresentaram risco de desnutrição.

Segundo Felix 2009, classificou 31,8\% das mulheres como desnutridas e $50 \%$ em risco de desnutrição, com resultado semelhante no grupo masculino, sendo $27 \%$ de homens desnutridos e $40 \%$ em risco de desnutrição, este estudo apresentou $75 \%(n=9)$ de mulheres com risco de desnutrição e $25 \%(n=3)$ com desnutrição. No sexo masculino foram encontrados $45,5 \%(n=5)$ com risco de desnutrição e número igual de pacientes desnutridos $(n=5)$.

O quadro de desnutrição no idoso tem apresentado tendência progressiva, devido a incidência de doenças e debilidades físicas associadas a idade. A deficiência nutricional e um agravante nessa população e o ocorre devido alterações no próprio envelhecimento, doenças sistêmicas que condicionam sem estado nutricional. (COLEMBERG, 2011)

A MAN aponta que idosos longevos tendem a apresentar mais desnutrição do que os de idade avançada, fato este diferente do encontrado no presente estudo, onde não houve diferença estatisticamente significante em relação a idade. No estudo de Colemberg, 2011, foram encontrados $14,72 \%$ de risco de desnutrição em pacientes com menos de 80 anos e 7\% apresentaram desnutrição com 90 anos ou mais.

Dados da população idosa brasileira segundo a pesquisa de orçamento familiar (IBGE 2003), separadas por sexo e faixa etária, mostraram déficit de peso em 8,9\% dos homens com 75 anos ou mais enquanto mulheres de ambas as faixas etárias (60-74 anos e 75 ou mais), não apresentaram déficit de peso. $O$ avançar da idade é um fator crucial para o aumento do número de idosos com desnutrição. Segundo Albuquerque 2009, a idade mostrou ser fator importante na diminuição dos valores de algumas variáveis, o que implica diretamente na necessidade de padrões de referência específicos para idosos.

\section{CONCLUSÃO}

Neste estudo observou-se que a maioria doa pacientes possuia menos de 70 anos, foi classificado como possível desnutrição no escore de controle, e na maioria confirmada desnutrição. Baseado nestes dados, verifica-se a importância de serem realizados mais estudos em relação a avaliação do estado nutricional em idosos, correlacionando com comorbidades, fatores socioeconômicos, hábitos alimentares, atividade física e o próprio conhecimento acerca de alimentação. Há também uma importância do clínico em aplicar a MAN em idosos internados, bem como ambulatoriais para avaliar os riscos e realizar intervenção precocemente, prevenir possíveis agravantes, diminuir o tempo de internação ou evitar a mesma, melhorar o prognostico do paciente e aumentar chance de recuperação.

\section{REFERÊNCIAS}

1. ABAJO CDC, GARCIA RS, CALABOZO FB, et al. Protocolo de valoración, seguimiento y actuación nutricional en un centro residencial para personas mayores. Nutr Hosp. 2008; 23:100-4.

2. BARBOSA-SILVA MCG, BARROS AJD. Avaliação nutricional subjetiva: Parte 1 - Revisão de sua validade após duas décadas de uso. Arquivos de Gastroenterologia, São Paulo, v. 39, n. 3, p.181-187, jul./set. 2002.

3. BATISTA RS. Nutrição e saúde, novas perspectivas. J Bras Med 1995;69(2):136-44. 
4. BLANCO VL, RAUSELL LG, VIDAL JV, et al. Nutritional assessment at the time of hospital-admission study initiation among different methodologies. Nutr Hosp. 2006; 21(2):163-172.

5. BRAGA D, SERRANO H. Perfil nutricional e socioeconômico de pacientes internados no Hospital e Maternidade Vital Brazil. Nutrir Gerais, Ipatinga, v. 1, n. 1, ago./dez. 2007.

6. CAMPOS MAG, PEDROSO ERP, LAMOUNIER JA, et al. Estado nutricional e fatores associados em idosos. Revista da Associação Médica Brasileira, São Paulo, v. 52, n. 4, p. 214-221, jul./ago. 2006.

7. CAMPOS MTFS, MONTEIRO JBR, ORNELAS APRC. Fatores que afetam o consumo alimentar e a nutrição do idoso. Revista de Nutrição, Campinas, v. 13, n. 3, p.157-165, set./dez. 2000.

8. DATASUS [Internet]. Brasília: Ministério da Saúde (BR) [cited 2010 sep 29]. Apresenta: Indicadores Demográficos segundo o IBGE e Sistema de Informações Hospitalares do SUS (SIH/SUS).

9. EMED TCXS, KRONBAUER A, MAGNONI D. Mini-avaliação nutricional como indicador de diagnóstico em idosos de asilos. Revista Brasileira de Nutrição Clínica, Porto Alegre, n.21, v.3, p.219-23, 2006.

10. FELIX LN, SOUZA EMT. Avaliação nutricional de idosos em uma instituição por diferentes instrumentos. Rev. Nutr., Campinas, v. 22, n. 4, p. 571-580, Aug. 2009.

11. GOMES RC, PINTO CS, SOAR C. Prevalência de desnutrição em idosos institucionalizados. Universidade do Vale do Paraíba - UNIVAP. São José dos Campos, SP, 2011. XIV Encontro Latino Americano de Iniciação Científica e Encontro Latino Americano de Pós-Graduação - Universidade do Vale do Paraíba.

12. GUIGOZ Y, VELLAS B, GARRY PJ. Assessing the nutrition status of the elderly: the mini nutritional assessment as part of the geriatric evaluation. Nutr Rev. 1996; 54(1 PT2): S59-65.

13. LIPSCHITZ DA. Screening for nutritional status in the elderly. Prim Care. 1994 Mar;21(1):55-67.

14. MARUCCI MFN, ALVES RP, GOMES MMBC. Nutrição na geriatria. In: Silva SMCS, Mura JDP. Tratado de alimentação, nutrição e dietoterapia. São Paulo: Roca; 2007. p. 391-416.Ministério da Saúde (BR), Secretaria de Atenção à Saúde, Departamento de Atenção Básica. Envelhecimento e saúde da pessoa idosa. Brasília: Ministério da Saúde 2006:8

15. MENEZES TN, MARUCCI MFN. Antropometria de idosos residentes em instituições geriátricas,Fortaleza, CE. Rev Saúde Pública. 2005; 39(2):169-75.

16. MICHELI ET, ABRAHÃO CLO, GRIGOLETTI SS, et al. Diagnóstico nutricional: comparação entre os instrumentos de avaliação nutrition risk screening (nrs-2002) e avaliação nutricional do Hospital de Clínicas de Porto Alegre (AN-HCPA). Rev HCPA. 2009;29(1):23-8.

17. MINAYO MCS, COIMBRA JUNIOR CEA. Antropologia, saúde e envelhecimento [online]. Rio de Janeiro: Editora FIOCRUZ, 2002. 209 p. ISBN: 85-7541-008-3.

18. SILVA MLT. Geriatria. In: Waitzberg DL. Nutrição oral, enteral e parenteral na prática clínica.São Paulo: Atheneu; 2000. p. 997-1008

19. SILVEIRA EA, KAC G, BARBOSA LS. Prevalência e fatores associados à obesidade em idosos residentes em Pelotas, Rio Grande do Sul, Brasil: classificação da obesidade segundo dois pontos de corte do índice de massa corporal. Cad. Saúde Pública, Rio de Janeiro, v. 25, n. 7, p. 1569-1577, July 2009. 